\title{
Indicadores cienciométricos y altmétricos para la identificación de líderes científicos
}

\begin{abstract}
Scientometric and altmetric indicators for scientific leaders' identification: the case of an Iberoamerican research academic network
\end{abstract}

Emilio Alberto Ortiz-Torres

Universidad de Holguin, Cuba

eortiz@uho.edu.cu

(iD http://orcid.org/0000-0002-0497-9063

Yoan Israel Viamonte-Garrido

Universidad de Holguin, Cuba

joanviamontes@gmail.com

(iD http://orcid.org/0000-0001-7974-6523

\section{Resumen:}

Hasta el presente, la literatura científica latinoamericana revisada, sobre el liderazgo científico universitario en las ciencias sociales ha ignorado, al menos en aquella publicada en plataformas bibliográficas gratuitas, el uso posible y necesario de indicadores cienciométricos, tradicionales y altmétricos, como parte de una metodología de identificación y evaluación temprana de dicho fenómeno en el contexto específico de las redes académicas iberoamericanas. El objetivo del presente trabajo es proponer una batería de 12 indicadores cienciométricos combinados con los cuales identificar y evaluar tempranamente el liderazgo científico en redes académicas latinoamericanas, empleando como fuentes de datos los perfiles de los investigadores objeto de estudio las plataformas académicas ResearchGate y Google Académico. Los indicadores propuestos fueron aplicados a un universo de 102 miembros de la Red Iberoamericana de Investigación sobre la Calidad de la Formación Doctoral en Ciencias Sociales, los cuales constituyen producto de la selección y la combinación de 6 indicadores cienciométricos tradicionales abordados por los autores en trabajos anteriores junto a otros 6 de tipo altmétricos obtenidos a partir de la producción científica de los investigadores en las plataformas académicas Research Gate y Google Académico. Como resultado de la presente investigación, 7 miembros de la Red fueron identificados como líderes científicos.

Palabras Clave: Indicadores cienciométricos y altmétricos, Líderes científicos latinoamericanos, Redes académicas iberoamericanas, Indicadores de ResearchGate, Indicadores de Google Académico.

\section{ABstract:}

So far, the revised Latin American scientific literature on university scientific leadership in the social sciences has ignored, at least in that published in for-free bibliographic platforms, the possible and necessary use of scientometric indicators, both traditional and altmetric ones as part of a methodology for an early identification and evaluation of such a phenomenon in the specific context of Ibero-American academic networks. The objective of the present paper is to propose a set of 12 combined scientometric indicators to identify and to evaluate early scientific leadership in the Latin American academic networks, by using as data sources the profiles of the researchers under study in the academic platforms ResearchGate and Google Scholar. The proposed indicators were applied to a universe of 102 members of the Ibero-American Research Network on the Quality of Doctoral Training in Social Sciences and constitute the product of the selection and combination of 6 traditional scientometric indicators addressed by the authors in previous works together with 6 other altmetric ones obtained from the scientific production of the researchers published on the academic platforms ResearchGate and Google Scholar. As a result of the present investigation, 7 members of the Network were identified as scientific leaders.

KEYWORDS: Scientometric and altmetric indicators, Latin American scientific leaders, Ibero-American research networks, ResearchGate indicators, Google Scholar indicators. 


\section{INTRODUCCIÓN}

El fenómeno del liderazgo, en su más general acepción, ha sido objeto de un gran número de investigaciones científicas desde inicios del siglo XX, con el aporte de variadas y ricas teorías desde disímiles enfoques, con énfasis y profundidad en sus características esenciales y su importancia social (Madachian, Noordin y Taherdoost, 2016). En este sentido, House y Aditya (1997) realizaron una revisión de las teorías contemporáneas más relevantes relacionadas con el liderazgo en términos genéricos, la cual les permitió identificar las contribuciones científicas importantes para el conocimiento de dicho fenómeno, así como aquellos aspectos fundamentales que ameritaban su continuidad investigativa.

Como bien afirma Contreras (2008), en términos generales, el liderazgo constituye un fenómeno complejo que ha sido entendido desde distintas aproximaciones teóricas, asociadas a las corrientes epistemológicas imperantes en un determinado momento y ha estado sujeto a los continuos cambios históricos y culturales gestados y desarrollados en la sociedad. La conceptualización del liderazgo ha generado un cuerpo de conocimientos un tanto ambiguo y transitorio, en tanto se modifica según los intereses de la sociedad que lo define y las tendencias teóricas vigentes. A este respecto, se concuerda con Castro, Flores, Cornejo y Castro (2019) en que es posible considerar al liderazgo como un sistema de competencias, dada la necesidad de considerar todas sus cualidades de manera integrada, aunque dichos autores lo valoren solo en el ámbito educativo.

Sin embargo, a diferencia de lo que sucede con otros tipos de liderazgos en los ámbitos políticos, educativos y comunitarios - sobre los cuales existe un cúmulo importante de aportes investigativos - el liderazgo científico en el contexto iberoamericano continúa siendo insuficientemente investigado. Si bien es cierto que este tipo específico presenta puntos de contacto comunes al resto de las categorías de liderazgo, dada la coexistencia de cualidades, atributos y entornos multidimensionales de trabajo compartido, en el liderazgo científico existen particularidades o especificidades que lo diferencian del resto en función de lógicas y dinámicas propias de su ethos y campo de actividad.

Con relación al liderazgo científico como objeto de estudio, varios investigadores lo han abordado desde diferentes perspectivas. Por una parte, Brad (2014) afirma que un atributo fundamental en la estructura axiológica del líder científico debe ser, ante todo, su creencia en el carácter beneficioso de la ciencia como agente de cambio y progreso para la sociedad. También expresa que este debe ser capaz de comunicar, irradiar y contagiar con su convicción sobre determinados objetivos, proyectos y resultados a un amplio auditorio, así como motivar mediante su visión, instruir mediante la lógica y concebir a la ciencia como un proceso creativo. Por otra parte, Brad (2014) considera que el líder científico es uno de los más necesitados por la sociedad, ya que guía e inspira a otros investigadores y estudiantes a partir de su sabiduría, valores e ideario, apoyados en el análisis objetivo de los datos, a partir de la lógica y de sus conclusiones fundamentadas en el método científico, sin obviar los aspectos subjetivos a los que están sujetos sus seguidores mientras los motiva y conduce, a la vez que investiga, escribe, enseña y ayuda a otros a resolver problemas.

Además, Antes (2018) destaca en primer lugar la esencia humana como prioritaria de la científica de los líderes académicos. En la misma línea, Pozenel (2018) resalta la importancia de las competencias académicas de éste en el quehacer científico, las cuales le permitirán dirigir proyectos en las organizaciones de investigación de una manera más efectiva y promotora de la innovación y la colaboración, mediante crecientes cuotas de compromiso espontáneo por parte de otros investigadores y de sus estudiantes.

Tradicionalmente, los líderes científicos en las instituciones universitarias - como su propia denominación lo indica- se han caracterizado por ir a la vanguardia de un determinado grupo académico de investigación, particularmente compuesto por doctores en ciencias de una o más áreas del conocimiento, con el objetivo común de conducirlos en la resolución de problemas científico-investigativos y docentes. A tal fin, dichos líderes exhiben una preparación y un desempeño superiores al resto de sus colegas con cuyo ejemplo o modelo logran orientar, aglutinar y encauzar los esfuerzos del resto de investigadores de su área académica en la 
obtención de resultados científicos novedosos y pertinentes, que a su vez los posicionan a ellos mismos y a sus instituciones a nivel local, nacional e internacional.

Por tanto, la evaluación del liderazgo científico a nivel institucional, nacional e internacional constituye una práctica universalmente aceptada en los sistemas de educación superior de todo el mundo. Numerosos autores se han se pronunciado sobre su pertinencia e importancia (Aguiar y Linares, 2019; Moñux, 2005; Villaveces, Orozco, Olaya, Chavarro y Suárez, 2005). En vista de la propia misión de las universidades, es imprescindible que su producción científica sea objeto de reflexión y evaluación debido a los imperativos del desarrollo social, y sobre ello igualmente diferentes autores se han referido (Agudelo et al, 2003; CortésVargas, 2007; García-Castrillo, Lafuente Felez, Montañes Bernal y Sanz Gracias, 1999).

Los estudios sobre el fenómeno del liderazgo científico en las universidades constituyen un reconocimiento como indicador de impacto internacionalmente aceptado en la evaluación, tanto del desempeño científicoinvestigativo y docente de individuos, instituciones y naciones. A tal fin, se emplean sistemas de matematización del conocimiento científico-tecnológico conocidos como estudios cienciométricos, que ofrecen a los evaluadores diversos índices obtenidos a partir de la aplicación de una amplia batería de indicadores.

En este contexto, Ortiz-Torres (2015) y Ortiz-Torres, González-Guitián, Infante-Pérez y ViamonteGarrido (2010) en investigaciones precedentes evidenciaron, como resultado científico colateral, la pertinencia de la aplicación de un grupo de indicadores cienciométricos a tesis doctorales en ciencias pedagógicas - así como a otras investigaciones educativas - que propiciaron la identificación de figuras de liderazgo entre una muestra aleatoria de investigadores con grado científico de doctor en relación a un universo de académicos de la Universidad de Holguín con igual graduación científica. Según dicho estudio, los líderes científicos identificados demostraron capacidad de convocatoria, labor aglutinadora y actividad orientadora que influía al resto de componentes de los grupos de investigación, a partir de su ejemplo personal de desempeño y sin que tuviesen que mediar necesariamente aspectos formales-institucionales como reglamentos internos, planes de trabajo, entre otros.

Por esta razón, los autores del presente artículo han concluido que, además de ser necesaria una conceptualización respecto a lo que es un líder científico, se precisa igualmente de la aplicación de un sistema de indicadores cienciométricos, tanto de actividad como de impacto, que permitan su identificación anticipada y ulterior desarrollo. En relación con la detección temprana de líderes científicos a través de indicadores bibliométricos, cabe destacar los aportes de Li (2009), para quien las redes bibliométricas, entendidas como las relaciones: autor-publicación, autor-lugar de publicación, publicación-publicación y de coautoría, sentaban las bases para evaluar tanto a líderes científicos consolidados, como a aquellos candidatos a líderes de alto potencial que aún no son considerados expertos pero que sin embargo se proyectan en esa dirección de acuerdo a su evolución en el tiempo y a los cuales llamó rising stars o "estrellas ascendentes".

Ante la insuficiencia y la alta dispersión de estudios científicos precedentes sobre la evaluación del fenómeno del liderazgo científico en las redes académicas iberoamericanas, a partir de la aplicación de indicadores cienciométricos específicos, en el presente trabajo se presentan de manera relativamente novedosa una propuesta metodológica construida a tal fin. La misma, obtenida a partir de un estudio de caso realizado en la Red Iberoamericana de Investigación sobre la Calidad de la Formación Doctoral en Ciencias Sociales, ${ }^{1}$ cuyos objetivos fueron identificar y evaluar el liderazgo científico presente en la referida red, sobre la base de los datos métricos públicamente accesibles desde las plataformas académicas de Google Académico y ResearchGate, lo cual se considera pertinente desde el punto de vista metodológico en los estudios sobre liderazgo científico (Thelwall y Kousha, 2017).

En Cuba, dentro del sistema de evaluación y acreditación de programas de doctorado de la Junta de Acreditación Nacional (2008) ${ }^{2}$ del Ministerio de Educación Superior (MES), en el patrón de calidad para la variable Impacto, se refiere explícitamente a la necesidad de determinar la existencia de líderes científicos 
en las instituciones de educación superior, como un indicador a aplicar a los doctores egresados de dichos programas. Además, el empleo de indicadores cienciométricos se encuentra fundamentado en el consenso, respecto a que la evaluación conjunta de la productividad científica y sus impactos constituye una necesidad estratégica de medición, como parte de la constatación del desarrollo integral de un país y no solo de las distintas áreas del conocimiento, de su política científico-tecnológica y de su gestión en función del desarrollo social y económico de cada nación.

Ortiz-Torres, González-Guitián, Infante-Pérez, Viamonte-Garrido y Reyes-Fernández (2013) ofrecieron por primera vez una definición de líder científico que se adopta en este artículo, según la cual se trata de aquel investigador cuya conjunción de atributos innatos, competencias adquiridas y visión vanguardista, le permiten asumir socialmente dicho rol en virtud del reconocimiento del mismo por sus pares académicos seguidores. El líder científico, además, es capaz de motivar a la vez que informa, agrupa, moviliza y guía a sus seguidores en la realización de proyectos de investigación para la obtención de resultados científicos pertinentes y novedosos, cimentados en su preparación académica, experiencia investigativa, aportes científicos, iniciativa, creatividad, activismo, desempeño superior, protagonismo, carisma y labor aglutinadora.

Como se constata en el concepto anterior, el liderazgo científico constituye un fenómeno científico y social, una síntesis original de cualidades personales innatas y adquiridas, que sumadas al binomio dialéctico autopercepción - percepción del liderazgo, se condensa en un rol social gracias al ejercicio sistemático de dichas facultades por parte del líder científico como profesor investigador en el entorno universitario. Es dicha actuación lo que lo posiciona ante sus seguidores como un modelo a seguir. En cada caso, un rasgo distintivo del líder científico consistirá en que casi siempre se le encontrará asociado al trabajo en equipo y la conducción de proyectos de investigación y casi nunca como investigador en solitario, lo cual se verá naturalmente reflejado en los análisis de coautorías de su producción científica en un periodo de tiempo.

Dado que el trabajo en equipo constituye en sí mismo un factor determinante dentro del fenómeno del liderazgo científico, el carisma que se aporta al desempeño investigativo en la actividad de colaboración académica se considera esencial, ya que es precisamente en esta síntesis valorativa donde emerge la identidad del líder científico. La identificación temprana de dichas figuras de liderazgo emergente en las universidades permitirá su ulterior desarrollo de manera propositiva y conveniente a las necesidades de la institución y de la sociedad.

Actualmente, la importancia de la identificación de líderes científicos en las universidades no solo posee un valor de uso en los marcos individuales de cada institución universitaria. La creciente globalización e internacionalización de la educación superior ha propiciado la creación de grupos y redes académicas de investigación en las cuales los líderes científicos son fundamentales y donde se precisan estudios que permitan su identificación temprana en pro de su ulterior desarrollo (Villamarín, 2011). En la historia del desarrollo científico universal es posible encontrar figuras académicas relevantes cuyos liderazgos contribuyeron notablemente al desarrollo de las ciencias de su pertenencia, en algunos casos como parte de redes académico-institucionales internacionales, una categoría reticular específica en la cual se enmarca la presente investigación.

Las redes de investigaciones científicas adquirieron una relevancia inédita durante la segunda mitad del siglo XX en los Estados Unidos y en algunos países europeos, impulsados ante todo por la revolución científica conocida entonces como Big Science o gran ciencia, una revolución científico tecnológica mayormente centrada en el uso y explotación de la energía atómica. A su vez, en el transcurso del siglo XXI las redes académicas constituyen formas novedosas de organización de la actividad científico-investigativa gracias al desarrollo en constante aceleración de las tecnologías de la información y de la comunicación. Allí la información científica, creada mediante la cooperación entre sus miembros, es puesta en circulación, transformada mediante la contextualización y la validación simultánea por el resto de la comunidad científica, a través de diversos ciclos de procesos de agregación de valor que desembocan en las múltiples tipologías de 
salidas de la producción científica que se conocen hoy. En la actualidad, la consolidación de estas redes es toda una realidad en la que se sustenta la colaboración científica de las instituciones de educación superior por todo el mundo, sin distingos del área del conocimiento que se busca desarrollar (Ramírez-Vidal, 2009).

Pérez, Román e Ibargollin (2018) consideran que la participación en redes, particularmente de tipo académico, se convierte en una fuente de riqueza y estímulo, tanto para docentes como investigadores y estudiantes, así como para sus instituciones y países de pertenencia, potenciando así el desarrollo de la educación superior al favorecer el espíritu de integración e interdependencia. Por tanto, las redes académicas internacionales constituyen un pilar fundamental en la búsqueda de la excelencia académica en la educación superior, a partir de la colaboración científica y tecnológica, intercambio de buenas prácticas y benchmarking de procesos, productos y servicios académicos que se innovan gracias a sus propias dinámicas. En este contexto, las redes académicas interuniversitarias nacionales o internacionales forman parte vital dentro de un marco diverso de funciones, estructuras y sistemas de la sociedad, así como también constituyen una forma de coordinación que combina la independencia con la interdependencia de los individuos y entes autónomos.

Al mismo tiempo, la importancia de la evaluación temprana del liderazgo científico en las redes académicas interuniversitarias nacionales e internacionales a través de indicadores tanto cienciométricos como altmétricos está dado en el natural interés de sus miembros o componentes en pertenecer a estructuras del conocimiento científico certificado con alto reconocimiento de sus pares nacionales e internacionales, el cual estará dado por la calidad de las aportaciones científicas de aquellos que lideran la red. Lo anterior constituye aún más una perentoria necesidad no satisfecha en América Latina particularmente dada la alta desigualdad socioeconómica presente en sus instituciones académicas, muchas de las cuales, como sucede en el caso cubano, están impedidas de acceder al conocimiento científico del mainstream internacional monopolizado por el norte global ya sea por razones económico-financieras, políticas o ambas. De ahí que el estudio del liderazgo científico temprano en redes académicas internacionales mediante las técnicas cienciométricas referidas permita, por una parte, a sus integrantes conocer quiénes de su membresía poseen un mayor potencial de contribución académica bilateral, así como a las ONGs que otorgan financiamiento a dichas redes detectar posibles beneficiarios de alto potencial científico futuro para el desarrollo científico de su país.

Varios estudios realizados sobre las redes académicas y científico-tecnológicas interinstitucionales de alcance internacional en Iberoamérica, han permitido clasificarlas en las siguientes tipologías: intergubernamentales, interuniversitarias nacionales e interuniversitarias internacionales. En lo que respecta a las redes académicas intergubernamentales, la experiencia iberoamericana presenta como estructuras reticulares pioneras de cooperación científica entre Estados, al Programa Iberoamericano de Ciencia y Tecnología para el Desarrollo (CYTED) y al Instituto Interamericano para la Investigación sobre el Cambio Global (IAI), siendo el primero concebido en España en los años ochenta del pasado siglo y el segundo en los EEUU en los años '90 del pasado siglo (Gual, 2014).

Ambas redes comenzaron como iniciativas de cooperación científica intergubernamental Norte-Sur impulsadas por los países del llamado norte global a nivel institucional y financiero. Finalmente, estas terminaron evolucionando hacia su conversión en redes más horizontales de cooperación sur-sur, en parte debido a la creciente estabilidad política y económica de América Latina durante la primera década del siglo XXI. El Programa CYTED liderado por España en esa época, tenía como misión constituirse en una plataforma para la diplomacia científica entre los Estados de la península ibérica y los de América Latina; un mecanismo de doble vía que respondiera, tanto a la colaboración entre los investigadores españoles y latinoamericanos, como al establecimiento de un nuevo modelo para la cooperación sur-sur entre países latinoamericanos con poca experiencia en el intercambio científico.

Algunas de las experiencias latinoamericanas exitosas en cooperación científica a través de redes interuniversitarias nacionales devenidas en internacionales, han sido valoradas por Serna, Castro y Calderón (2019), quienes realizaron un estudio de caso donde se analizan las dinámicas reticulares de colaboración 
interuniversitarias en Colombia en el área de administración. En dicha evaluación se emplearon sólo indicadores de actividad científica y se excluyeron la evaluación de indicadores de impacto. La red académica anterior resultó ser de tipo interinstitucional de alcance nacional y no tuvo en cuenta el liderazgo científico de sus investigadores, sino solo el institucional.

La idoneidad de la selección de las plataformas académicas Google Académico y ResearchGate y sus métricas para la evaluación temprana tanto de los candidatos con potencial de liderazgo científico como de sus producciones, ha sido validada por diversos autores (Harzing y Alakangas, 2016; Moed, Barllan y Halevi, 2016; Orduña-Malea, Martín-Martín y Delgado, 2016). Las conclusiones derivadas de un estudio comparativo relevante entre las plataformas académicas de Internet Google Académico, ResearchGate, Scopus y la $W e b$ of Science, que las dos primeras ofrecen más temprana y eficientemente las citaciones como indicador de impacto sobre las últimas dos. Si bien la "velocidad" de publicación no constituye un factor de impacto fundamental, estos autores consideran que su superioridad subyace en que tanto Google Académico como ResearchGate permiten más eficiente y oportunamente obtener altmétricas de liderazgo que la $W o S$ y $S c o p u s$ en una línea temporal.

De acuerdo a los autores antes citados, la eficiencia de Google Académico sobre Scopus y la $W o S$ para la realización de dicha evaluación, se evidencia en la considerable demora de las últimas plataformas para la publicación en sus bases de datos de los artículos aprobados. Es así como el estudio concluyó que este es un problema recurrente que presentan las citas tradicionales, en vista de que las mismas suelen tomar años desde la etapa de producción científica hasta su publicación en las revistas. En contraste, Google Académico resulta una alternativa lógica porque sus índices pueden explotar eficientemente los documentos web, pese a que la recolección manual de los datos puede consumir mucho tiempo.

Con relación a Research Gate, dichos autores consideran que ésta es la plataforma profesional más usada por científicos de las ciencias sociales, las artes y las humanidades, aunque en todos los casos Google Académico es más popular para todas las ciencias. De modo que ambos autores concluyeron en su estudio que existen suficientes evidencias empíricas para afirmar que la visibilización de las publicaciones científicas en estas plataformas académicas de Internet contribuye a atraer más citas sobre los trabajos allí indexados o publicados, según sea el caso, que en otras plataformas. En Research Gate, desde el punto de vista estadístico, se encontraron significativamente muchas menos citas que en Google Académico, pero a su vez muchas más que en Scopus y la Web of Science juntas en un período de tiempo.

El objetivo de la Red Iberoamericana de Investigación sobre la Calidad de la Formación Doctoral en Ciencias Sociales es coordinar y divulgar las actividades académicas y de producción científica que realizan sus investigadores, en pro de la elevación de la calidad en la formación doctoral en ciencias sociales en las instituciones miembros, así como potenciar el desarrollo de innovaciones educativas conjuntas (Ortiz-Torres y Romero-Rodríguez, 2018). Está compuesta por 102 investigadores procedentes de 40 universidades de 11 países iberoamericanos, la cual presenta la siguiente distribución de su membresía por origen nacional: Argentina (13), Chile (3), Colombia (3), Costa Rica (1), Cuba (63), Ecuador (3), España (6), México (5), Nicaragua (1), Perú (3) y Puerto Rico (1).

\section{MÉtodos, materiales EMPleAdos y FUentes}

El estudio de caso que se presenta en este trabajo se realizó entre septiembre de 2019 y febrero de 2020. Para la selección de la muestra se asumió el criterio de representatividad a partir de un universo de 126 miembros de la red procedente de 40 universidades de 11 países iberoamericanos. Posteriormente, se seleccionaron aleatoriamente 3 investigadores de cada universidad, cuya membresía a la red se ubicaba en el rango de entre 5 y 29 investigadores ( 8 universidades reunieron este criterio y de ellas se seleccionaron aleatoriamente 21 investigadores). Por otra parte, fueron incluidos en la muestra la totalidad de universidades que contaban entre 1 y 3 miembros en la red ( 3 universidades, $n=34$ ). La sumatoria de los 
dos segmentos de análisis anteriores permitió obtener una muestra inicial de 55 investigadores, lo cual representaba el 53,92\% del universo estudiado. Los datos de los investigadores estudiados fueron recopilados entre el 15 de noviembre y el 28 de enero de 2020.

Esta muestra fue posteriormente discriminada a través de los siguientes filtros de selección: 1) Número de doctores en ciencias (los doctorandos fueron descartados); 2) Productividad científica en el periodo 2009-2019; y 3) Disponibilidad de perfiles académicos publicados en las plataformas Google Académico y ResearchGate. Tras la referida selección de datos la composición de la muestra disminuyó de 55 a 26 $(-55 \%)$, debido a que 28 doctores en ciencias, con un mínimo de 10 años de productividad científica, presentaban las siguientes limitaciones: a) no contaban con perfiles académicos en Internet; b) presentaban sus perfiles académicos en plataformas académico-empresariales de Internet como LinkedIn o Academia.edu exclusivamente; c) contaban con un solo perfil, sea en Google Académico o en ResearchGate, lo cual impedía obtener las métricas complementarias de manera integral. A fin de preservar el anonimato de los investigadores y evitar sesgos perceptivos a lo largo del proceso de evaluación, a cada una de las 26 unidades muestrales les fue asignado un código alfanumérico como identificador. Si bien los presentes autores reconocen una muestra de solo 26 investigadores $(20,6 \%)$ entre un universo de 126 miembros de la red objeto de estudio (100\%), las limitaciones que impidieron que la muestra fuese mayor se explican en el apartado "Resultados".

El carácter inédito que los autores le confieren al presente trabajo consiste en la obtención de una batería única de 12 indicadores cienciométricos y altmétricos combinados orgánicamente a partir de la conciliación entre las dinámicas e indicadores propios del fenómeno del liderazgo científico y de las fuentes de recolección de datos en Internet. En la Tabla 1 "Indicadores cienciométricos y fuentes de datos aplicados al estudio del liderazgo científico en la Red" se presenta cada uno de los indicadores presentados sobre los 12 indicadores cienciométricos y altmétricos combinados que se establecieron para la evaluación del liderazgo científico en la Red así como las plataformas de Internet que sirvieron como fuentes de los datos en relación a los investigadores estudiados. Subsiguientemente, se presenta la fundamentación metodológica para el uso de cada uno de los indicadores cienciométricos propuestos, tanto los 6 previamente empleados por los presentes autores como aquellos 6 obtenidos a partir del uso de las plataformas académicas digitales propuestas por Thelwall y Kousha (2017), antes citados. 
TABLA 1

Indicadores cienciométricos y fuentes de datos aplicados al estudio del liderazgo científico en la red

\begin{tabular}{|l|l|}
\hline Indicadores Cienciométricos & Fuente de Datos \\
\hline 1- No. Proyectos Científicos & Google Académico \\
\hline 2- No. De Proy. Autoría Principal & Google Académico \\
\hline 3- Índice de Public. Autor Principal & Google Académico \\
\hline 4- Coeficiente Inverso Autorías en Solitario & Google Académico \\
\hline 5- Índice de Interés & ResearchGate \\
\hline 6- Índice de Citaciones & Google Académico + ResearchGate \\
\hline 7- Índice h & Google Académico \\
\hline 8- Índice i10 & Google Académico \\
\hline 9- No. de Seguidores Académicos & ResearchGate \\
\hline 10- Factor de Internacionalización & Google Académico + ResearchGate \\
\hline 11- No. Ponencias Eventos Nac. e Internac. & Google Académico \\
\hline 12- Total de Lecturas ResearchGate & ResearchGate \\
\hline Factor de Liderazgo Científico & No. Ind. Acum./ No. Ind.Evaluados \\
\hline
\end{tabular}

Fuente: elaboración propia.

- Número de proyectos cientificos: total de publicaciones científicas indexadas/publicadas en las que el investigador ha participado, independientemente de su rol como autor principal o primer coautor. Si la evaluación se realiza externamente y sin entrevistar al investigador evaluado, se considera válido asumir para este indicador que cada artículo científico diferente publicado en las plataformas Google Académico y Research Gate equivale a un proyecto, si bien en la práctica cada proyecto podría conducir a más de un artículo en un periodo de tiempo. Este indicador no debe incluirse en el cálculo del Factor de liderazgo científico por constituir un número base para calcular los restantes índices.

- Número de proyectos cientificos como autor principal: total de proyectos/artículos producidos por el investigador en la calidad de primer autor. Este indicador no debe emplearse en el cálculo del Factor de liderazgo científico por constituir un número base para calcular los restantes índices.

- Índice de publicaciones como autor principal: resulta el cociente derivado de los dos indicadores básicos anteriores, al dividir el número de proyectos científicos como autor principal entre el total de publicaciones científicas. Se obtiene un decimal ubicado entre 0 y 1 , entre más cerca de 1 se encuentre el investigador, mayor peso relativo de liderazgo científico tendrá.

- Coeficiente inverso de autorías en solitario: se obtiene como porcentaje inverso del número de artículos publicados en solitario, respecto al total de artículos publicados en un periodo de tiempo. Se basa en la lógica de que el número y porcentaje de autorías en solitario será inversamente proporcional trabajo en equipo, al llevar implícito la ausencia de equipos de investigación en la gestión científica del investigador estudiado. De este modo, si el porcentaje de autorías en solitario es del $10 \%(0,10)$ su coeficiente inverso es $90 \%(0,90)$.

- Índice de interés: refleja el grado general de interés que suscitan las publicaciones científicas de cada investigador en el resto de la comunidad científica de 15 millones de miembros de todo el orbe, presentes en la plataforma ResearchGate. Se obtiene a partir de la métrica Total Research Interest de dicha plataforma académica. Su medición precisa una actualización sistemática de los perfiles de los investigadores objeto de estudio en esta plataforma. 
- Índice de citaciones: dado el error estadístico a que conduciría la sumatoria de las citaciones en ambas plataformas (ResearchGate y Google Académico) debido al posible solapamiento de las citas, solo se tomarán de una u otra plataforma el número mayor de citaciones obtenidas por cualquiera de las dos plataformas. Resulta esencial que los perfiles académicos de los investigadores estudiados se encuentren creados y actualizados en ambas plataformas.

- Índice h: se centra en el núcleo de las publicaciones más citadas mostrando que $\mathrm{n}$ artículos han sido citados $n$ veces. Es considerado más confiable en la plataforma académica Google Académico que en ResearchGate (Hirsch, 2007).

- Índice i10: se parece al Índice h pero si el i10 es igual a 1, establece que el investigador cuenta con 10 artículos citados al menos 10 veces y así sucesivamente. Es provisto exclusivamente por la plataforma académica Google Académico.

- Número de seguidores académicos: se refiere al número de seguidores académicos según las métricas de Research Gate al tiempo de la consulta a la plataforma.

- Factor de internacionalización: se obtiene por la sumatoria de diferentes autorías principales y coautorías interinstitucionales internacionales, derivadas de los proyectos científicos/artículos en que el investigador estudiado, en representación de sí mismo, de su institución y su país, ha participado y publicado en un periodo de tiempo. El Factor de internacionalización de un investigador será directamente proporcional a su liderazgo científico nacional e internacional. Resulta un indicador cienciométrico reticular inédito. Si bien la mera participación en una red académica internacional o el número de proyectos internacionales en sí mismo no constituye un indicador de liderazgo científico, el número de proyectos científicos internacionales liderados por dicho investigador en el contexto de una red académica internacional, medida que se obtiene a partir del número total y porcentaje de publicaciones científicas derivadas como autor principal o primer coautor, sí constituye un indicador de liderazgo dentro de la red.

- Número de ponencias nacionales e internacionales presentadas en eventos académicos nacionales e internacionales en un periodo de tiempo.

- Total de Lecturas en ResearchGate: constituye una medida de visibilidad de la producción científica del investigador estudiado. Research Gate propicia de manera única en su plataforma este indicador al tiempo en que se realiza la consulta sobre el investigador evaluado.

Resultante del cálculo secuencial y acumulativo de los indicadores anteriores, se obtiene una media aritmética a la que se denominó Factor de liderazgo científico. El mismo no constituye uno de los 12 indicadores propuestos, sino que resulta ser solo un factor de posicionamiento o ranking para las unidades muestrales estudiadas en base al coeficiente obtenido por éstas en cada uno de los 12 indicadores propuestos. Tras el cálculo del factor de liderazgo obtenido para cada investigador, aquellos cuyos coeficientes que se ubican en el primer cuartil constituirán el núcleo duro del liderazgo real o potencial de una red académica dada, con independencia de su naturaleza, sea esta intergubernamental, interuniversitaria nacional o internacional.

Para la recolección de los datos se accedió a los perfiles de cada una de las unidades muestrales y se realizó la importación de datos manualmente. La etapa de análisis de los datos se llevó a cabo con el paquete de programas de Microsoft Office, particularmente Microsoft Excel 2013 para el análisis y Microsoft Word 2013 para la redacción de las inferencias derivadas del estudio. Como se ha mostrado antes, las fuentes de los datos analizadas han sido exclusivamente los perfiles académicos de los investigadores estudiados públicamente, accesibles en Internet en las plataformas académicas Google Académico y ResearchGate. En ningún modo la presente investigación ha obtenido ni empleado datos que violen la privacidad de los 26 investigadores estudiados o de alguna fuente distinta. 


\section{Resultados}

Como se adelantó en el apartado "Métodos, materiales empleados y fuentes”, el presente estudio presentó como limitación el hecho de que, si bien originalmente se pensó aplicar la batería de indicadores cienciométricos y altmétricos propuestos a entre un $50 \%$ y un $100 \%$ de miembros de la red objeto de estudio, dando por sentado en ellos una cultura generalizada del uso de las plataformas que ocupan el presente trabajo se ha podido constatar que apenas el 20,6\% de estos, o sea, 26 investigadores, contaban con perfiles académicos disponibles tanto en Google Académicos como en ResearchGate. Los presentes autores reconocen que si bien estadísticamente la muestra aludida es insuficiente para considerarla generalizable, aun así el resultado en sí mismo permitió a los autores identificar este punto débil en la membresía de la Red con vistas a futuras acciones de promoción de una cultura del uso eficiente y oportuno de las plataformas académicas y sus altmetrías junto a los indicadores cienciométricos tradicionales en los procesos de evaluación del liderazgo científico universitario.

Amén de lo anterior, el presente estudio logró el objetivo de identificar a 7 investigadores líderes con grado científico de doctor en ciencias en la red, los cuales constituyen el 6,86\% del total de investigadores que componen el universo y aproximadamente el $27 \%$ de la muestra evaluada. Su liderazgo científico en cada caso se calculó a partir de la aplicación de 12 indicadores cienciométricos propuestos, tanto de actividad como de impacto. La determinación del liderazgo científico en la Red se realizó procurando obtener de la tabulación y análisis de los datos importados de las redes académicas de Internet Google Académico y ResearchGate, un ordenamiento o ranking de los resultados de cada investigador, tanto por cada indicador evaluado, como de manera integral.

La evaluación integral de la muestra estudiada fue agrupada en el primer cuartil muestral de mayor a menor aquellos investigadores cuyas coocurrencias estadísticas exhibieron los mayores valores por indicador, estableciendo, por tanto, como líderes científicos a aquellos doctores cuya presencia en el primer cuartil de los resultados acumulaban un mínimo de 7 y un máximo de 10 indicadores para el presente estudio. Finalmente, la cantidad total de indicadores acumulados se dividía entre el total de indicadores evaluados, para de este modo obtener un Coeficiente de Liderazgo Científico expresado en decimales y ubicado entre 0 y 1 , de modo que entre más cercano a 1 resulte el valor obtenido, mayor sería el factor de liderazgo científico en cada una de las unidades muestrales de la investigación.

En la Figura 1 titulada "Dispersión de los coeficientes de liderazgo científico en la red" se presentan, en el Eje de las X el valor del Coeficiente de Liderazgo Científico en la Red obtenido por cada uno de los 26 investigadores estudiados, mientras que en el Eje de las Y se representa a cada uno de estos representados con códigos alfanuméricos. Como puede apreciarse, en dicha figura aparecen representadas las posiciones relativas de cada una de las unidades muestrales evaluadas, a partir de su Coeficiente de liderazgo científico y donde se puede apreciar en 19 de los menores coeficientes una elevada dispersión que tiende a reducirse notablemente en el caso de los 7 líderes identificados. Igualmente, como se ha señalado antes, existen valores extremos en todos los indicadores evaluados en los casos de los investigadores J1 y E3, quienes presentan valores atípicos para el resto de las unidades muestrales en 10 de los 12 indicadores evaluados. 
FIGURA 1

Dispersión de los coeficientes de liderazgo científico en la red

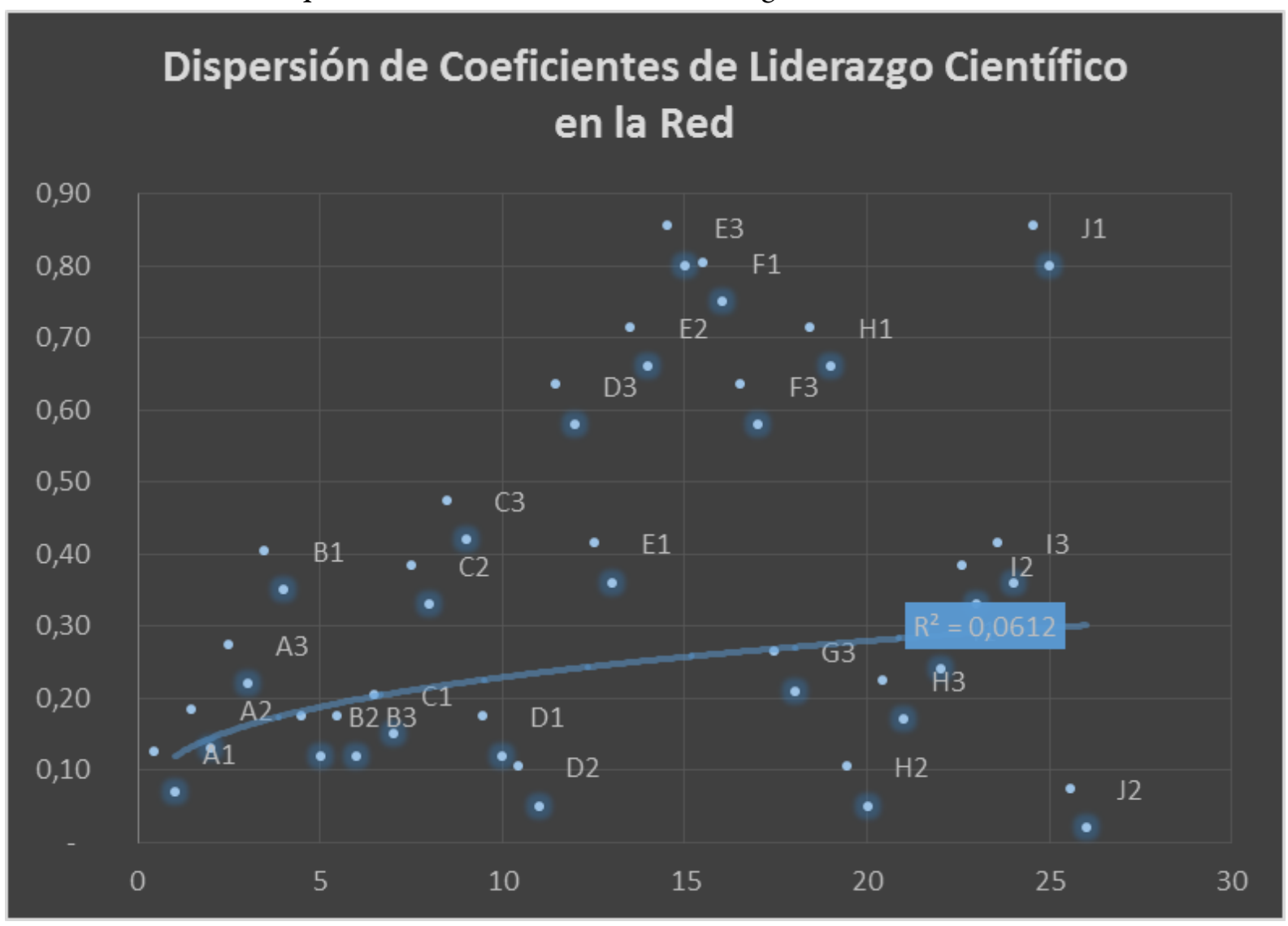

Fuente: elaboración propia.

A fin de ilustrar la atipicidad y los valores extremos exhibidos tan solo por el investigador J1, sirva de muestra que el mismo, para el periodo de tiempo estudiado de 2009 a 2019, presentó una productividad científica de 872 artículos, de los cuales el $41 \%$ resultaron de su autoría principal. Respecto al grado de trabajo en equipo manifestado por dicho investigador líder, obtuvo un coeficiente inverso de autorías en solitario del $82 \%$, lo cual es una medida directamente proporcional a una elevada productividad científica derivada del trabajo en equipo. Al mismo tiempo, respecto a los indicadores de impacto para el mismo investigador, en el periodo estudiado de 10 años recibió un total de 12761 citas. A fin de profundizar en el grado de análisis sobre la cantidad de artículos científicos más citados del investigador J1, se evaluaron complementariamente sus Índices h e i10, los cuales resultaron ser de 76 y 391 respectivamente, evidenciando así no solo una alta productividad científica, sino también un elevado índice de citaciones, ya que contaba con 76 publicaciones que contabilizaban 76 citas cada una y 391 artículos que contaban con al menos 10 citas cada uno. Asimismo, el investigador J1 contabiliza en ResearchGate un total de 881 seguidores académicos, un Factor de internacionalización de 71, 211 ponencias en eventos nacionales e internacionales y un Número de lecturas de sus investigaciones de 157060 durante el periodo de tiempo evaluado.

Asimismo, el presente estudio también permitió determinar una línea de tendencia cuyo coeficiente de determinación $\mathrm{R}^{2}=0,0612$ permite inferir un desarrollo casi nulo del Coeficiente de liderazgo científico en la Red, ya que ninguno de los doctores evaluados acumuló una posición de liderazgo en el primer cuartil en el 100\% de los indicadores aplicados.

Sin embargo, pese a que ninguna de las 26 unidades muestrales alcanzó una posición de liderazgo para el primer cuartil de resultados en el 100\% de los indicadores evaluados, sí es significativo que al menos en 7 
de los 26 investigadores estudiados (26,92\%), se encontraran en posiciones relativas de liderazgo, al alcanzar presencia en el primer cuartil en el ordenamiento de los resultados, para un total de entre 7 y 10 de los 12 indicadores cienciométricos de liderazgo científico empleados. Se considera que aquellos 4 investigadores (H1, E2, D3 y F3) que obtuvieron un Coeficiente de liderazgo entre 0,58 y 0,66, podrían ser considerados líderes científicos potenciales, que al ser tempranamente identificados y desarrollados podrían mejorar su desempeño en los indicadores deficientes. Por su parte, se sugiere que como líderes científicos reales se tomen sólo a aquellos 3 investigadores (J1, E3 y F1), cuyo Coeficiente de liderazgo científico se encontró en el rango de valores de entre $0,75-0,80$.

TABLA 2

Ranking del Liderazgo científico en la red

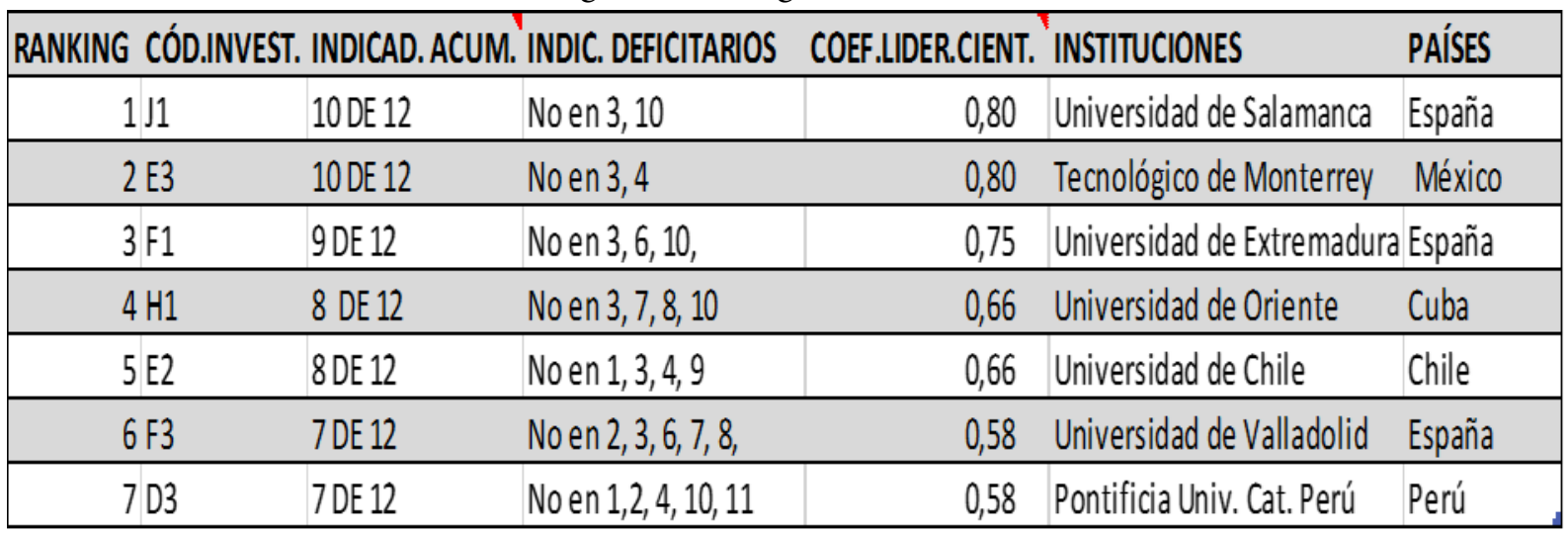

Fuente: elaboración propia

Después de haber destacado los resultados globales más relevantes obtenidos, se presentan de manera analítica los datos más pertinentes con relación a cada uno de los indicadores evaluados. En relación al primer indicador cienciométrico, Número de proyectos científicos, los valores encontrados para todas las unidades muestrales estudiadas, estuvieron entre 3 y 872 en un periodo de 10 años (2009 - 2019). Debe significarse, sin embargo, que el rango de este indicador se ubicó entre 55 y 872 para los 7 líderes científicos encontrados.

Durante la investigación resultó muy interesante encontrar que los mismos dos valores extremos correspondían exclusivamente a los dos primeros investigadores J1 (872 proyectos/artículos) y E3 (300 proyectos/artículos). Cabe destacar que dichas cifras de publicaciones científicas son de 3 a 8 veces superiores al valor máximo encontrado en el rango de los restantes 6 líderes científicos identificados, que en el caso del investigador I3 va de un mínimo de 55 proyectos/artículos a un máximo de 121 proyectos/artículos del doctor F3 para el periodo estudiado.

El resto de 19 investigadores estudiados (el 73\% de la muestra) presentaron un rango entre 3 y 52 proyectos/artículos para el periodo de tiempo establecido. Se considera que la causa de tan bajos valores para la mayor parte de la muestra de doctores estudiados, podría no deberse necesariamente a una baja productividad científica, sino a una ineficiente actualización de sus perfiles académicos en las plataformas analizadas por parte de los investigadores analizados. Este indicador resulta crítico para la evaluación del liderazgo científico en las redes académicas porque los tres indicadores cienciométricos que le siguen en el presente análisis constituyen variables dependientes del mismo.

Dado que los indicadores Número de artículos como autor principal e Índice de publicaciones como autor principal, se encuentran correlacionados con el primer indicador y ambos son proporcionales entre sí, se centrarán los resultados en este último. El estudio realizado mostró una alta dispersión de datos en los valores para este indicador, que resultan tan bajos como 0,13 , pasando por valores medios de 0,41 para alcanzar un máximo de 0,89 , considerado como muy alto y tomando como referencia a 1 como valor ideal. Lo anterior permite inferir que, para los 7 líderes científicos encontrados en la red, era más importante el volumen de la 
productividad científica en términos globales, alcanzada generalmente a través de una elevada frecuencia de colaboraciones como coautores, que como resultado de salidas de proyectos conducidos por ellos mismos. Igualmente llamó la atención que solo hubo un investigador cuyo valor para el indicador objeto de estudio se ubicó excepcionalmente en 1, significando que en todo su volumen de producción científica resultó el conductor de cada uno de los proyectos en que participó y por ende el autor principal de cada uno de ellos.

Sin embargo, el volumen de producción científica de este investigador para el periodo de tiempo estudiado de 10 años fue de apenas 14 proyectos/artículos. Cuando este investigador fue evaluado en el resto de indicadores, tampoco rebasó los 5 indicadores de liderazgo de un total de 12. Al mismo tiempo, resultó significativo encontrar un grupo de 8 investigadores, distintos al de los 7 líderes científicos antes identificados, que si bien presentaban valores de medios a altos para este indicador (entre 0,47 y 0,83), tampoco reunían volúmenes globales de producción científica significativos ni evidenciaron liderazgo en el resto de los indicadores evaluados. Por tal razón, a los componentes de dicho grupo no se les pudo considerar líderes científicos de manera integral.

Respecto al indicador Coeficiente inverso de autorías en solitario, las 26 unidades muestrales presentaron cifras que oscilaban de un mínimo de $0 \%$ a un máximo de $80 \%$. En lo relacionado con los 7 líderes científicos identificados en la red, sus valores se posicionaban entre un mínimo de $8 \%$ a un máximo de 36\% para este indicador, lo cual evidencia que generalmente estos investigadores obtuvieron una tasa de interacción con sus equipos de investigación de entre un $64 \%$ a un $92 \%$ en la gestión de sus publicaciones científicas y como reflejo directo de su trabajo en equipo, ya sea con otros colegas investigadores o con sus estudiantes tutorados.

En el caso de 6 investigadores de la muestra analizada (61\%), llamó la atención que presentaron valores ubicados entre $47 \%$ y $80 \%$ de autorías en solitario, lo cual indica que su tasa de trabajo en equipos científicos en el periodo de tiempos estudiado estuvo entre el $53 \%$ y el $20 \%$, lo cual expresa valores que van de medios a muy bajos de interacción investigativa-colaborativa. La evaluación de este indicador se considera relevante porque no se conciben líderes científicos sin equipos de investigación y este novedoso indicador permite, por primera vez, evaluar las coautorías como medida de trabajo investigativo en equipo. La inferencia más importante que ofrece este indicador es que el Porcentaje de autorías en solitario será inversamente proporcional al factor de Liderazgo científico.

El indicador Índice de Interés constituye una novedad por parte de la plataforma académica ResearchGate, pues fue incorporada a las métricas de dicho portal desde febrero de 2019. Este nuevo indicador tiene como objetivo medir la influencia de un documento y un autor a partir de la integración sintética de distintas métricas, tanto tradicionales: citas recibidas, como altmétricas: lecturas y recomendaciones (Delgado y Orduña-Malea, 2019).

El comportamiento del presente indicador establece de manera natural un ranking basado exclusivamente en el nivel de interés que, tanto un autor como sus publicaciones como un todo o en partes, suscita dentro de un universo de más de 180 millones de publicaciones científicas que dicha plataforma custodia. En el caso de las 26 unidades muestrales del presente estudio sus índices de interés oscilaban de un mínimo de 0 a un máximo de 5196.

En el 77\% de la muestra estudiada sus Índices de Interés de ResearchGate se posicionaban entre 0 y 37 , mientras en el 23\% de los casos restantes dicho indicador obtuvo valores entre 87,6 y 5196,0 pertenecientes a aquellos 7 investigadores considerados como líderes potenciales y reales. En relación a estos últimos, resultó relevante constatar que los investigadores J1 y E3 lideraban el ranking conformado al exhibir valores extremos para todos los indicadores aplicados respecto al resto de la muestra estudiada. En relación al 77\% de investigadores que no resultaron identificados como líderes tras la evaluación, cuyos valores usualmente resultaron sorprendentemente bajos, los autores asumen que la causa principal pudo deberse más a una falta de actualización sistemática de sus perfiles en las plataformas académicas digitales que a una deficiencia real en su producción científica en sí, aunque tampoco se descarta en ciertos casos esta última causa. 
Hasta el momento, los indicadores antes analizados corresponden a los indicadores de productividad científica (Spinak, 1996). Sin embargo, la evaluación del liderazgo científico-docente también debe incluir como condición sine qua non la evaluación a partir de indicadores de impacto científico como los siguientes:

Los Índices de citaciones, Índice h e Índice i10 constituyen indicadores de impacto por excelencia y se encuentran fuertemente correlacionados entre sí. Estas métricas se encuentran disponibles en Google Académico desde 2005 para cada uno de los millones de investigadores que cuentan con un perfil público en dicha plataforma, dentro del apartado Google Académico Citaciones, así como para cualquier actor ajeno que deseen consultarlos.

A este respecto, las consultas formuladas a esta plataforma sobre determinado investigador ofrecerán dichos tres Índices y su evolución en el tiempo. De este modo Google Scholar permite evaluar, tanto el total de citaciones que las publicaciones de un investigador han obtenido en el tiempo, como cuáles de dichas publicaciones han generado los mayores impactos en término de citas.

Los Índices h e i10 permiten discriminar aún más lo que lo hacen las citaciones, al permitir al evaluador constatar de entre un número de publicaciones citadas, cuáles han sido en efecto las de más impacto, pues el Índice h permite calcular el nivel de impacto de un autor, revista, institución o país, a partir de cuántos artículos X han recibido X citas cada uno en un periodo de tiempo. En el caso del Índice i10 calcula la misma correlación para aquellas publicaciones que cuenten con al menos 10 citas cada una (Universidad de Castilla La Mancha, 2013).

La aplicación de estos tres indicadores a las unidades muestrales del presente estudio permitió apreciar en el Índice de Citaciones valores que se encontraban en un rango de entre 0 y 12761 . En relación a los Índices h estos se comportaron con valores entre 0 y 76, mientras los Índices i10 se ubicaron entre 0 y 391.

De manera similar a la evaluación de los indicadores anteriores, estos tres índices correlacionados entre sí permitieron identificar un grupo de 7 doctores en ciencias que lideraron este indicador. Si bien en distintos puestos del ranking para este indicador, los 7 investigadores que destacaron en el mismo corresponden a los mismos 7 antes identificados. Los Índices h de los 7 doctores líderes se ubicaron entre 10 y 76 mientras los índices i10 para este grupo se estableció entre 10 y 391.

De acuerdo con Hirsch, creador del Índice h, en las ciencias de la vida se considera que un investigador, tras 20 años de productividad científica y que presente un Índice h de 20, se considera normal, uno con 40 sobresaliente y uno de 60 o más simplemente excepcional (Díaz, 2014). Sin embargo, en las ciencias Sociales a día de hoy no existen marcos de referencia respecto a qué valores se consideran normales, extraordinarios y excepcionales, por lo cual este trabajo hará uso de los valores antes determinados por Hirsch.

De modo que, atendiendo al periodo de tiempo analizado, los 7 líderes identificados en el presente estudio exploratorio presentan un comportamiento que se ubica entre los valores normales a lo excepcional, este último en tan solo una de las unidades muestrales. Los Índices i10 en los dos primeros investigadores que encabezan el ranking de los 7 líderes científicos identificados en la red, fueron de 391 y 97 respectivamente, significando que cada uno de estos contaban con al menos 10 publicaciones que habían recibido 391 y 97 citas cada una respectivamente.

En lo relacionado con el indicador Número de seguidores académicos, se consideró su inclusión como indicador debido a que no se concibe un líder sin seguidores. En el caso de otras formas de liderazgo como el político-mediático - los llamados influencers - estas métricas llevan años en uso, no siendo el caso del liderazgo académico en el cual, hasta el momento, dicho indicador no se encontraba sistematizado en la literatura especializada pese a que la plataforma Research Gate contabiliza el número de seguidores académicos de cada investigador que posee un perfil en dicho portal.

En el caso de las 26 unidades muestrales del presente trabajo exploratorio, este indicador se ubicó en valores que oscilan entre 0 y 881 . Resultó notable, al mismo tiempo, que los 7 investigadores que lideraron el ranking en los indicadores antes analizados, resultaron los mismos que contaban con el mayor número de Seguidores académicos, donde el valor mínimo fue de 58 seguidores y el máximo de 881 . Vale precisar 
que estos seguidores son exclusivamente aquellos de entre la comunidad académica que se hospeda en la plataforma Research Gate y no contabiliza los seguidores en otras plataformas, como Twitter debido a que ResearchGate constituye, en tanto plataforma académica, un filtro positivo como discriminante.

Dado que las redes académicas internacionales llevan implícito un proceso de internacionalización, se decidió construir el Factor de internacionalización. Como se ha expresado antes, dicho factor se calcula obteniendo la sumatoria de las diferentes autorías principales y como primer coautor en que haya participado el investigador estudiado, donde cada investigador extranjero diferente constituye una unidad representativa por país extranjero diferente que conforma su red académico-colaborativa particular. Solo se contabiliza el número total de diferentes nacionalidades de coautorías con los cuales el investigador estudiado ha publicado en un periodo de tiempo, como medida de cooperación académica materializada en diferentes proyectos científicos/artículos en representación de sí mismo, de su institución y de su país. El Factor de internacionalización de un investigador será directamente proporcional a su liderazgo científico nacional e internacional, especialmente cuando se trate de cooperación académica Sur-Norte, aunque tal grado de especificidad no formó parte de los objetivos del presente estudio.

El número de Ponencias en eventos nacionales e internacionales también se consideró un indicador útil en la identificación de líderes científicos, dado que se caracterizan, entre otros aspectos, por un marcado interés en socializar los resultados de sus investigaciones en foros nacionales e internacionales de alto impacto para su comunidad científica. Respecto a este indicador, los valores de los 26 investigadores objeto de estudio se ubicaron entre 0 y 211 en el periodo de tiempo analizado. Sin embargo, la brecha valorativa entre los 7 líderes científicos identificados y el resto de las unidades muestrales, salvo en el caso del investigador principal de dicho ranking, a diferencia de los indicadores antes evaluados, no es muy amplia, ya que estos oscilaron entre 5 y 20 en el caso de los restantes 6 líderes científicos. Lo anterior permite apreciar que la divulgación de los resultados de las investigaciones era preferiblemente realizada desde las revistas científicas u otros formatos, que en eventos académicos. Sin embargo, el hecho de que el principal líder científico identificado obtuviera un valor tan elevado de participaciones en estos foros indica que no pierden relevancia como indicador.

Como último indicador cienciométrico a aplicar en la evaluación del liderazgo científico en la Red, se decidió incluir, como medida el Índice de visibilidad de los trabajos investigativos de la muestra estudiada, la afluencia del tráfico académico en Internet sobre un determinado investigador y sus publicaciones. A tal fin, se empleó el indicador Número de lecturas de Research Gate. Cabe destacar que los resultados de la aplicación de este indicador resultaron coherentes con obtenidos en los anteriores. Los valores exhibidos por los 26 doctores evaluados se establecieron entre 0 y 157060 lecturas. Debe aclararse que este valor, a diferencia de otros indicadores antes analizados, no se toma en cuenta a lo largo del periodo estudiado de 10 años, sino solo respecto al comportamiento de este indicador al momento de realizar la consulta.

En el análisis cienciométrico y altmétrico combinado realizado fue posible identificar a los 7 líderes científicos de la Red, establecer un ranking del liderazgo científico institucional iberoamericano y determinar en cuáles indicadores dichos líderes mostraron deficiencias y deberían trabajar por perfeccionar, ya que en ningún caso se pudo establecer el liderazgo en la totalidad de los indicadores evaluados.

Como se puede apreciar en la Tabla 2, el liderazgo científico en la red objeto de estudio corresponde con países generalmente desarrollados o industrializados - con la excepción de Cuba- y cuyas publicaciones científicas a su vez se ven beneficiadas por unas políticas de ciencia, tecnología e innovación, generalmente incentivadoras de una alta productividad científica individual. Igualmente, durante el estudio fue posible detectar una fuerte correlación colaborativa y citativa entre las dos instituciones y países que encabezan el ranking antes mostrado, exhibiendo la existencia de un colegio invisible entre la Universidad de Salamanca y el Instituto Tecnológico de Monterrey que contribuyó al apalancamiento mutuo en los valores extraordinarios encontrados, tanto en los indicadores de productividad científica como de impacto. 


\section{Conclusiones}

Como el liderazgo científico en las instituciones de educación superior se encuentra insuficientemente investigado en el contexto latinoamericano, a pesar de su importancia y pertinencia en los momentos actuales, se han elaborado varios indicadores cienciométricos para su identificación y evaluación temprana, a partir de los datos aportados por las plataformas académicas Google Académico . ResearchGate y su validación parcial mediante un estudio de caso en una red académica internacional.

Los indicadores cienciométricos propuestos se consideran pertinentes, factibles de utilizar y con un valor de uso para los estudios sobre líderes científicos en las ciencias sociales en las redes académicas latinoamericanas. El carácter parcial de dicha validación empírica evidencia la necesidad de continuar con su aplicación a otras redes, así como de aquellos indicadores cienciométricos que no fue posible aplicar por falta de información de los investigadores de la muestra, lo que constituye una limitación de este estudio y hace necesaria su continuidad investigativa para una mayor certeza científica de los resultados aportados.

\section{Agradecimientos}

Los autores reconocen el apoyo financiero del Ministerio de Educación Superior de la República de Cuba, para la realización del proyecto de investigación Gestión de la calidad de las tesis doctorales en ciencias pedagógicas a través de un sistema de indicadores para evaluar su impacto cientifico en las condiciones de masividad de la nueva universidad cubana (código RED-03), del que se han derivado varios resultados científicos parciales, uno de los cuales lo constituye el contenido del presente artículo. El proyecto fue dirigido por Emilio Alberto Ortiz Torres y participaron como miembros: María Virginia González Guitián, Inés Infante Pérez, Yoan Israel Viamonte Garrido y Nelvys Reyes Fernández.

\section{REFERENCIAS}

Agudelo, D.; Bretón-López, J.; Ortiz-Recio, G.; Poveda-Vera, J.; Teva, I.; Valor-Segura, I. y Vico, C. (2003). Análisis de la productividad científica de la psicología española través de las tesis doctorales. Psicothema, 15(4), 595-609. Recuperado de http://www.psicothema.com/pdf/1113.pdf

Aguiar, J. D. y Linares, M. P. (2019). Los estudios relacionados con la producción científica: apuntes teóricos conceptuales. Anales de la Academia de Ciencias de Cuba, 9(2). Recuperado de http://www.revistaccuba.cu/in dex.php/revacc/article/view/638

Antes, A. (2018). First law of leadership: be human first, scientist second. Nature, 563(601). Recuperado de https:// www.nature.com/magazine-assets/d41586-018-07530-7/d41586-018-07530-7.pdf

Brad, G. (29 de enero de 2014). The three types of leaders the world needs most: artistic, scientific and interpersonal. Forbes. Recuperado de https://www.forbes.com/sites/georgebradt/2014/01/29/the-three-types-of-leaders-the -world-needs-most-artistic-scientific-and-interpersonal/\#b3c6bf63c489

Castro, S. A.; Flores, F.; Cornejo, C. A. y Castro, M. A. (2019). Competencias de liderazgo que movilizan docentes en puestos directivos escolares de establecimientos educativos particulares subvencionados de la $V$ Región de Chile. Revista educación, 43(2). Recuperado de https://www.scielo.sa.cr/pdf/edu/v43n2/2215-2644-edu-43-02-0020 1.pdf

Contreras, F. (2008). Liderazgo: perspectivas de desarrollo e investigación. International Journal of psychological research, 1(2), 64-72. Recuperado de http://www.redalyc.org/articulo.oa?id=299023508008

Cortés-Vargas, D.(2007). Medir la producción científica de los investigadores universitarios: la bibliometría y sus límites. Revista de la educación superior, 36(142), 43-65.

Delgado, E. y Orduña-Malea, E. (2019). Research interest score: el nuevo indicador bibliométrico que mide la influencia de las publicaciones de un autor en ResearchGate. Recuperado 
de https://www.researchgate.net/publication/331673341_Research_interest_score_el_nuevo_indicador_bibl iometrico_que_mide_la_influencia_de_las_publicaciones_de_un_autor_en_ResearchGate

Díaz, G. J. (2014). El Índice H: una forma objetiva de evaluar la producción científica de investigador. Revista de la Facultad de Medicina Veterinaria y de Zootecnia, 61(2), 13-114. Recuperado de http://www.scielo.org.co/scielo .php?script $=$ sci_arttext\&pid=S0120-29522014000200001\&lng=en\&tlng=es

García-Castrillo, P.; Lafuente Félez, A.; Montañés Bernal, A. y Sanz Gracias, F. (1999). Producción científica en economía según publicaciones en revistas internacionales: el caso de España. Papeles de economía española, 81, 49-57.

Gual, M. (2014). El papel de las redes científicas intergubernamentales en las relaciones regionales y la integración de América Latina. Science \& diplomacy, 3(4). Recuperado de: http://www.sciencediplomacy.org/article/2015/elpapel-de-las-redes-cientificas-intergubernamentales-en-las-relaciones-regionales-y

Harzing, A. W. y Alakangas, S. (2016). Google Scholar, Scopus and the Web of Science: a longitudinal and crossdisciplinary comparison. Scientometrics, 106(2), 787-804.

Hirsch, J.E. (2007). Does the h index have predictive power?. Proceedings of the National Academy of Sciences of the United States of America, 104(49), 19193-19198. Recuperado de https://www.ncbi.nlm.nih.gov/pmc/articles /PMC2148266/

House, R. J. y Aditya, R. N. (1997). The social scientific study of leadership: quo vadis? Journal of management, 23(3), 409-473. Recuperado de http://psgleadership.scripts.mit.edu/2008IAP/pdf/1_required_reading/Scientific\%2 0views\%20on\%20Leadership.pdf

Li, X.-L.; Foo, C.S.; Tew, K.-L. y Ng, S.-K. (2009). Searching for rising stars in bibliography networks. En International Conference on Database Systems for Advanced Applications.pp. 288-292). Berlin: Springer-Verlag. Recuperado de https://dl.acm.org/doi/10.1007/978-3-642-00887-0_25

Madachian, M.; Noordin, F. y Taherdoost, H. (2016). Leadership theories: an overview of early stages. Recent advances in energy, environment and financial science, 198(201). Recuperado de https://www.researchgate.net/publicatio n/305323677_Leadership_Theories_an_Overview_of_Early_Stages

Moed, H.; BarIlan, J. y Halevi, G. (2016). A new methodology for comparing Google Scholar and Scopus. Journal of informetrics, $10(2), 533-551$.

Moñux, D. (2005) Condicionantes políticos y problemas metodológicos en la evaluación de impacto social de las políticas de I+D e innovación. Revista CTS, 4(2).

Orduña-Malea, E.; Martín-Martín, A. y Delgado, E. (2016). The next bibliometrics: ALMetrics (Author Level Metrics) and the multiple faces of author impact. El profesional de la información, 25(3), 485-496.

Ortiz-Torres, E. A.; González-Guitián, M. V., Infante-Pérez, I. y Viamonte-Garrido, Y. I. (2010). Evaluación del impacto científico de las tesis doctorales en ciencias pedagógicas mediante indicadores cienciométricos. Revista española de documentación cientifica, 33(2), 279-286. doi: https://doi.org/10.3989/redc.2010.2.728

Ortiz-Torres, E. A. González-Guitián, M. V.; Infante-Pérez, I. Viamonte-Garrido, Y. I. y Reyes-Fernández, N. (2013). La identificación de los líderes científicos en la Universidad de Holguín. Pedagogía universitaria, 18(3), 24-33. Recuperado de http://cvi.mes.edu.cu/peduniv/index.php/peduniv/article/download/576/pdf_1

Ortiz-Torres, E. A.; González Guitián., M. V.; Infante-Pérez, I. y Viamonte-Garrido, Y. I. (2015). La evaluación del impacto científico en las investigaciones educativas a través de un estudio de caso. Revista electrónica de investigación educativa, 17(2), 89-100. Recuperado de https://redie.uabc.mx/redie/article/view/422/1226

Ortiz-Torres, E. A. y Romero-Rodríguez, R. (2018). Red Iberoamericana sobre la calidad de la formación doctoral en ciencias sociales: experiencias y perspectivas. Ciencia e innovación tecnológica, 67(2), 2881-2886.

Pérez, Y.; Román, E. e Ibargollín, W. (2018). Las redes académicas como necesidad y oportunidad de superación del claustro universitario. Pedagogía y sociedad, 21(53), 258-278. Recuperado de http://revistas.uniss.edu.cu/index .php/pedagogia-y-sociedad/article/view/733

Pozenel, M. (2018). The importance of leadership skills in the scientific workforce. Recuperado de https://www.cas.org/ blog/importance-leadership-skills-scientific-workforce 
Ramírez-Vidal, J. A. (2009). Redes académicas de educación e investigación. Recuperado de http://www.ai.org.mx/ai/a rchivos/coloquios/7/Redes\%20Academicas\%20de\%20Educacion\%20e\%20Investigacion.pdf

Serna, M. F.; Castro, E. S. y Calderón, G. (2019). Las redes de colaboración en el área de administración: el caso de las universidades colombianas. Revista española de documentación cientifica, 42(2), 236-252. doi: https://doi.or $\mathrm{g} / 10.3989 /$ redc.2019.2.1595

Spinak, E. (1996). Diccionario Enciclopédico de bibliometría, cienciometría e informetría. Paris: UNESCO. Recuperado de https://unesdoc.unesco.org/ark:/48223/pf0000243329

Thelwall, M. y Kousha, K. (2017). ResearchGate vs. Google Scholar: which finds more early citations?. Scientometrics, 112(2), 1125-1131. doi: http://doi.org/10.1007/s11192-017-2400-4

Universidad de Castilla La Mancha (2013). Indices de citas y factor de impacto e indicios de calidad en publicaciones académicas para procesos de acreditación y sexenios. Recuperado de https://www.educacion.to.uclm.es/pdf/inves tigacion/citas_factor-de-impacto.pdf

Villamarín, M. G. (2011). Redes académicas y producción de conocimiento pertinente. Hallazgos, 8(16), 43-62. Recuperado de https://revistas.usantotomas.edu.co/index.php/hallazgos/article/download/1681/1830

Villaveces, J. L.; Orozco, L. A.; Olaya, D. L.; Chavarro, D. y Suárez, E. (2005). ¿Cómo medir el impacto de las políticas de ciencia y tecnología? Revista iberoamericana de ciencia, tecnología y sociedad, 2(4), 125-146.

\section{Notas}

1 La Red Iberoamericana de Investigación sobre la Calidad de la Formación Doctoral en Ciencias Sociales, en lo adelante referida como la Red, es auspiciada y financiada por la Asociación Universitaria Iberoamericana de Posgrado (AUIP) y coordinada desde la Universidad de Holguín, Cuba, oficialmente aprobada por la AUIP el 20 de octubre de 2016 y posee su página web oficial en la dirección: https://riidoctoralcs.net/

2 Si bien la Junta de Acreditación Nacional de la República de Cuba no cuenta con un sitio web propio, sírvase consultar los indicadores de calidad de la misma a través del siguiente enlace del Ministerio de Educación Superior de la República de Cuba: https://www.mes.gob.cu/es/indicadores-de-calidad 